\title{
Ion-Solvent Interactions in Acetylacetone I. Free Energies of Transfer of the Silver Ion and its Complex Formation Constans with Other Solvents
}

\author{
Ikko SAKAMOTO* and Satoshi OKAZAKI ${ }^{\dagger}$
}

Received September 16,1987;Accepted October 31,1987

Acetylacetone (2,4-pentanedione, Hacac) is one of $\beta$-diketones, which has widely been used as a chelating reagent. It is a protic and less basic solvent of a relatively high dielectric constant $\left[27.2\right.$ at $25{ }^{\circ} \mathrm{C}$ (1)]. In the previous papers (2-4), we studied the dissociation equilibria of 1-1 electrolytes in Hacac by conductometry and reported the results as follows.

(1) The ion association constants of alkali metal salts decrease in Hacac with the increase in crystallographic radii of cations. This cation dependence of the ion association constants is consistent with that in acetone, while in acetonitrile and alcohols the ion association constants increase with increasing cation sizes. The solvating ability of Hacac towards cations, that is, the basicity of Hacac is weaker than that of acetonitrile and alcohols.

(2) The anion dependence in Hacac of the ion association constants of tetraalkylammonium halides and the limiting molar conductivities of halide ions is similar to that in alcohols which can interact effectively with anions through hydrogen bonding. This anion dependence in Hacac is in contrast with that in acetone, which indicates the hydrogen bonding ability of Hacac based on the enol form of the solvent molecules.

(3) The homoconjugation constants of weak acids in Hacac are nearly equal to those in aprotic solvents such as acetonitrile and propylene carbonate. The intramolecular hydrogen bonding in the enol form of Hacac appreciably reduces the solvating ability towards anions, that is, the acidity of the solvent as well as the basicity. In this connection, the autoprotolysis constant $K$, of Hacac is rather small $\left[\mathrm{pK}_{\mathrm{s}}=19.3(3)\right]$. Acetylacetone is a less basic and less acidic amphiprotic solvent.

In this communication have been determined potentiometrically the free energies of transfer of the silver ion from Hacac to such solvents as acetonitrile (AN), acetone (AC), water, methanol $(\mathrm{MeOH})$, ethanol (EtOH), dimethylformamide (DMF), dimethylacetamide (DMA) and dimethylsulfoxide (DMSO). The emf of the following cell (i) was measured at $25^{\circ} \mathrm{C}$.

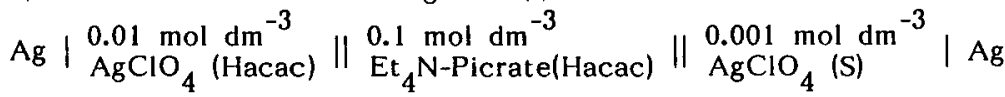

In the cell (i), $S$ denotes Hacac and other solvents. The liquid junction potential between the salt bridge of tetraethylammonium picrate solution in Hacac and a silver perchlorate solution in a solvent $S$ was assumed to be negligibly small (5). The complex formation constants of the silver ion in Hacac with other solvents were also determined from the emf changes of the cell (ii) upon the addition of a solvent $S$.

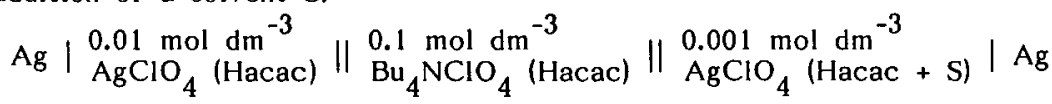

The complex formation equilibrium of the silver ion in Hacac with other solvents added to the cell (ii) is expressed by eqns. (iii) and (iv).

$$
\begin{aligned}
& \mathrm{Ag}^{+}+\mathrm{nS}=\mathrm{Ag}^{+}(\mathrm{S})_{\eta} \\
& \beta_{n}=\left[\mathrm{Ag}^{+}(\mathrm{S})_{n}\right] /\left(\left[\mathrm{Ag}^{+}\right][\mathrm{S}]^{\mathrm{n}}\right)
\end{aligned}
$$

Here, $\mathrm{Ag}^{+}$is the silver ion solvated only by Hacac, $n$ is the number of solvating $S$ molecule(s), and $\beta_{n}$ is the over-all complex formation constant. The methods and assumptions discussed in detail $^{n}$ by Izutsu et al. $(6,7)$ were used for the determination of the complex formation constants.

The transfer free energies and the complex formation constants of the silver ion are summarized in Tale 1. Acetylacetone is the weakest solvent to solvate the silver ion among the solvents in Table 1. The transfer free energies obtained in this study are compared in Fig. 1 with those

Department of Chemistry, Faculty of Education, Shimane University, Matsue 690

† Department of Chemistry, Faculty of Science, Kyoto University, Kyoto 606

Key Words: Acetylacetone, Silver Ion Solvation, Transfer Free Energy 
Table 1. Over-all complex formation constants $\left(\beta_{n}\right)$ of the silver ion in acetylacetone (Hacac) with other solvents, $(S)$ and the free energies of ${ }^{n}$ transfer of the silver ion from Hacac to $\mathrm{S}\left(\Delta \mathrm{G}^{\mathrm{o}}{ }_{\mathrm{tr}} / \mathrm{kcal} \mathrm{\textrm {mol } ^ { - 1 }} ; 1 \mathrm{cal}=4.184 \mathrm{~J}\right)$.

\begin{tabular}{|c|c|c|c|c|c|}
\hline Solvent (DN) & $\beta_{1}$ & $\mathrm{~B}_{2}$ & $\beta_{3}$ & $\beta_{4}$ & $\Delta G^{o}{ }_{t r}$ \\
\hline Hacac $(-)$ & - & - & - & - & 0 \\
\hline AN $(14.1)$ & 34 & 320 & 710 & - & -8.4 \\
\hline$(17.0)$ & 1.3 & - & - & - & -2.2 \\
\hline $\mathrm{H}_{0} \mathrm{O} \quad(18.0)$ & 4.3 & 5.5 & 9.1 & - & -4.0 \\
\hline MeOH $(19.0)$ & 2.0 & 0.3 & - & - & -2.1 \\
\hline $\mathrm{EtOH}(20.0)$ & 2.1 & 2.1 & - & - & -2.9 \\
\hline DMF $\quad(26.6)$ & 18 & 36 & 140 & - & -7.7 \\
\hline DMA $(27.8)$ & 38 & 310 & 1800 & - & -9.6 \\
\hline DMSO $(29.8)$ & 110 & 160 & 16000 & 35000 & -12.4 \\
\hline
\end{tabular}

$\mathrm{DN}$; donor number, $\mathrm{AN}$; acetonitrile, $\mathrm{AC}$; acetone, $\mathrm{DMF}$; dimethylformamide,

DMA; dimethylacetamide, DMSO; dimethylsulfoxide.

reported in the literature; the agreement is fairly good. Referring to Table 1, only monosolvated silver ions were formed for $\mathrm{AC}$, while mono- and di-solvated ions were formed for $\mathrm{MeOH}$ and EtOH. Tri-solvated species were also detected for AN, water, DMF and DMA. For DMSO, a complexing up to four solvent molecules is observed. The complexing of the silver ion in Hacac becomes easier with the increase of the basicity of the solvent added in the order of $\mathrm{AC}, \mathrm{MeOH}, \mathrm{EtOH}<\mathrm{DMF}$ < DMA \& DMSO. This order is consistent with that of stability of the silver ion in pure solvents. Hydration is stronger than expected from the donor number of water (8). The abnormally strong complexing of the silver ion with $\mathrm{AN}$ is attributed to the back bonding interaction of the silver ion with the nitrile group (9). The linear relation is observed between the $\log \beta_{1}$ (or $\log \beta_{\mathrm{n}}$ ) values and the transfer free energies, which indicates that the ion - solvent interactions in the primary coordination sphere play an important role in the ionic solvation. The correlation between the observed values of transfer free energies and the calculated values based on the coordination model of ionic solvation proposed by Cox et al. (10) will be discussed later.

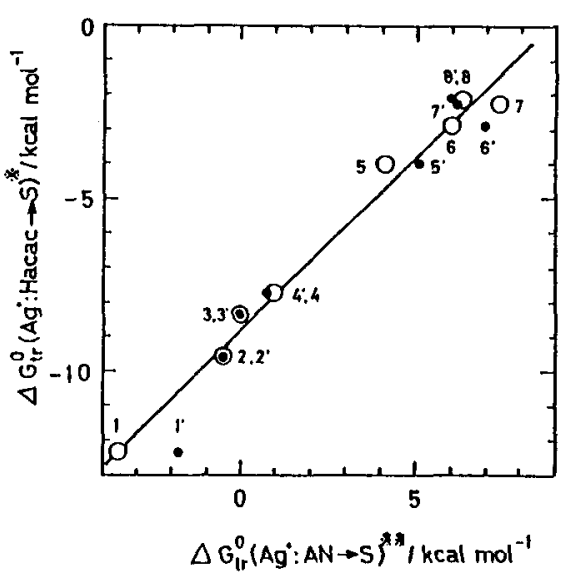

Fig. I Relation between the free energies of transfer of the silver ion obtained in this work and those in the literature.

*; this work, **; Ref. 5 [O, 1-8 (negligible liquid junction potential method) and $O, I^{\prime}$ $-8^{\prime}$ (tetraphenylarsonium tetraphenylborate reference electrolyte method)], 1, 1'; DMSO, 2,2'; DMA, 3,3'; AN, 4,4'; DMF, 5,5'; water, 6,6'; EtOH, 7,7'; acetone, 8,8'; MeOH. Slope $=1$.

\section{REFERENCES}

1) T. Fujinaga, S. Okazaki, I. Sakamoto, K. Masuda and H. L. Lee, Bunseki Kagaku, 29, T24(1980).

2) I. Sakamoto, K. Masuda, S. Okazaki and T. Fujinaga, Electrochim. Acta, 26, 197(1981).

3) I. Sakamoto, K. Masuda, S. Okazaki and T. Fujinaga, Bunseki Kagaku, 31, E49(1982).

4) I. Sakamoto and S. Okazaki, Denki Kagaku, 51, 117(1983).

5) R. Alexander, A. J. Parker, J. H. Sharp and W. E. Waghorne, J. Amer. Chem. Soc., 94,1148(1972).

6) K. Izutsu, T. Nomura, T. Nakamura, H. Kazama and S. Nakajima, Bu11. Chem. Soc. Jpn, 47, 1657 (1974).

7) K. Izutsu, T. Nakamura and K. Iwata, Ana1. Chim. Acta, 117, 329(1980).

8) V. Gutmann, "Coordination Chemistry in Non-aqueous Solutions", Springer-Verlag, Wien, p.19 (1968).

9) B. G. Cox, G. R. Hedwig, A. J. Parker and D. W. Watts, Aust. J. Chem., 27, 477(1974).

10) B. G. Cox, A. J. Parker and W. E. Waghorne, J. Phys. Chem., 78, 1731(1974). 